\title{
Being Licked by a Dog Can Be Fatal: Capnocytophaga canimorsus Sepsis with Purpura Fulminans in an Immunocompetent Man
}

\author{
Naomi Mader ${ }^{1}$, Fabian Lührs ${ }^{1}$, Stefan Herget-Rosenthal ${ }^{1}$, Martin Langenbeck ${ }^{2}$ \\ ${ }^{1}$ Department of Medicine, Rotes Kreuz Krankenhaus, Bremen, Germany \\ ${ }^{2}$ Department of Emergency and Intensive Care Medicine, Rotes Kreuz Krankenhaus, Bremen, Germany
}

Received: 09/09/2019

Accepted: 23/09/2019

Published: 09/10/2019

\begin{abstract}
How to cite this article: Mader N, Lührs F, Herget-Rosenthal S, Langenbeck M. Being licked by a dog can be fatal: capnocytophaga canimorsus sepsis with
\end{abstract} purpura fulminans in an immunocompetent man. EJCRIM 2019;6: doi:10.12890/2019_001268.

Conflicts of Interests: The Authors declare that there are no competing interest

This article is licensed under a Commons Attribution Non-Commercial 4.0 License

\section{ABSTRACT}

Bite infections caused by Capnocytophaga canimorsus are rare. Severe and fatal infections are more frequently reported in patients with immunodeficiency, splenectomy or alcohol abuse. We describe the case of a 63-year-old man who developed flu-like symptoms and presented after some delay with severe sepsis and purpura fulminans. He was found to be infected with $C$. canimorsus without a bite injury and did not demonstrate immunodeficiency or any other typical predisposition. Despite extensive intensive care, his conditions deteriorated and he died from multiorgan failure.

\section{LEARNING POINTS}

- Pet owners with banal, for instance flu-like, symptoms should urgently seek medical advice when symptoms are unusual.

- Capnocytophaga canimorsus infection should be considered and empirical antibiotic therapy immediately started or adjusted in the presence of purpura fulminans in the absence of animal bites or immunodeficiency.

\section{KEYWORDS}

Capnocytophaga canimorsus, immunocompetent, purpura fulminans, sepsis

\section{CASE DESCRIPTION}

A 63-year-old previously healthy man presented with a 3-day history of fever and progressive dyspnoea. The day before he had developed facial petechiae, dysaesthesia of his right lower limb and myalgia of the lower extremities. He had been touched and licked, but not bitten or injured, by his dog, his only pet, in previous weeks. There was no hospitalisation or recent travel abroad. On examination, in addition to petechiae there were ecchymoses over the lower limbs but no open wounds. The patient was conscious and febrile $\left(39^{\circ} \mathrm{C}\right)$, with dyspnoea at rest and a respiratory rate of $36 / \mathrm{min}$, hypoxic (percutaneous oxygen saturation level $70 \%$ ) and anuric but haemodynamically stable. He

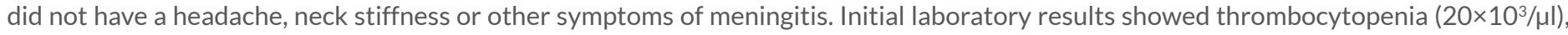
lymphocytopenia $\left(0.2 \times 10^{3} / \mu \mathrm{l}\right)$, elevated procalcitonin $(>100 \mathrm{ng} / \mathrm{ml})$ and CRP $(205 \mathrm{mg} / \mathrm{l})$. The partial thromboplastin time was $>180 \mathrm{~s}$, and the International Normalized Ratio was 3.51. The patient had acute kidney injury (creatinine $3.4 \mathrm{mg} / \mathrm{dl}$, BUN $116 \mathrm{mg} / \mathrm{dl}$ ) and signs of liver dysfunction (AST $438 \mathrm{U} / \mathrm{I}$, ALT $142 \mathrm{U} / \mathrm{I}, \mathrm{GGT} 991 \mathrm{U} / \mathrm{l}$, bilirubin $3.96 \mathrm{mg} / \mathrm{dl}$ ) as well as rhabdomyolysis (creatine kinase $1556 \mathrm{U} / \mathrm{l}$ ). Arterial blood gases revealed lactic acidosis ( $\mathrm{pH} 7.217$, lactate $12.8 \mathrm{mmol} / \mathrm{I})$. After the patient was referred to the ICU, an initial diagnosis of severe sepsis with purpura fulminans was made and he was immediately treated with clarithromycin and piperacillin/tazobactam to cover Streptococci, Neisseria meningitidis, Haemophilus influenza and Staphylococcus aureus, and with ceftriaxone due to the differential diagnosis of leptospirosis. 
A CT scan of the abdomen and thoracic cavity showed no sign of infection or pathological conditions.

The patient's condition deteriorated over the next 30 hours. He developed encephalopathy and paralytic ileus. Purpura and renal and liver failure progressed (Figs. 1 and 2). He experienced a cardiac arrest, but was successfully resuscitated, and then intubated and mechanically ventilated. Severe hypotension persisted, so we proceeded with norepinephrine (0.03 $\mu \mathrm{g} / \mathrm{kg} / \mathrm{h})$, hydrocortisone (200 mg/24 h), dobutamine $(0.125 \mu \mathrm{g} / \mathrm{kg} / \mathrm{h}$ ) and bicarbonate. The patient received erythrocyte ( 8 units) and platelet ( 2 units) concentrates, and 8 units of fresh frozen plasma. We initiated haemodialysis.

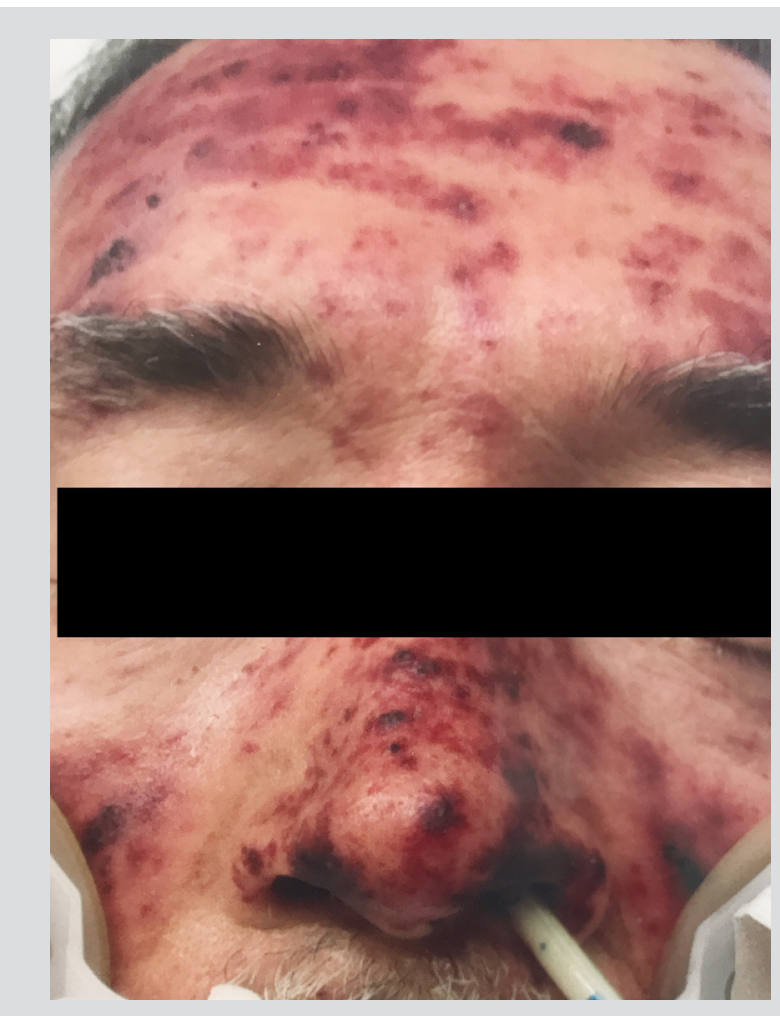

Figure 1. Patient's face with purpura fulminans unchanged a few days after admission

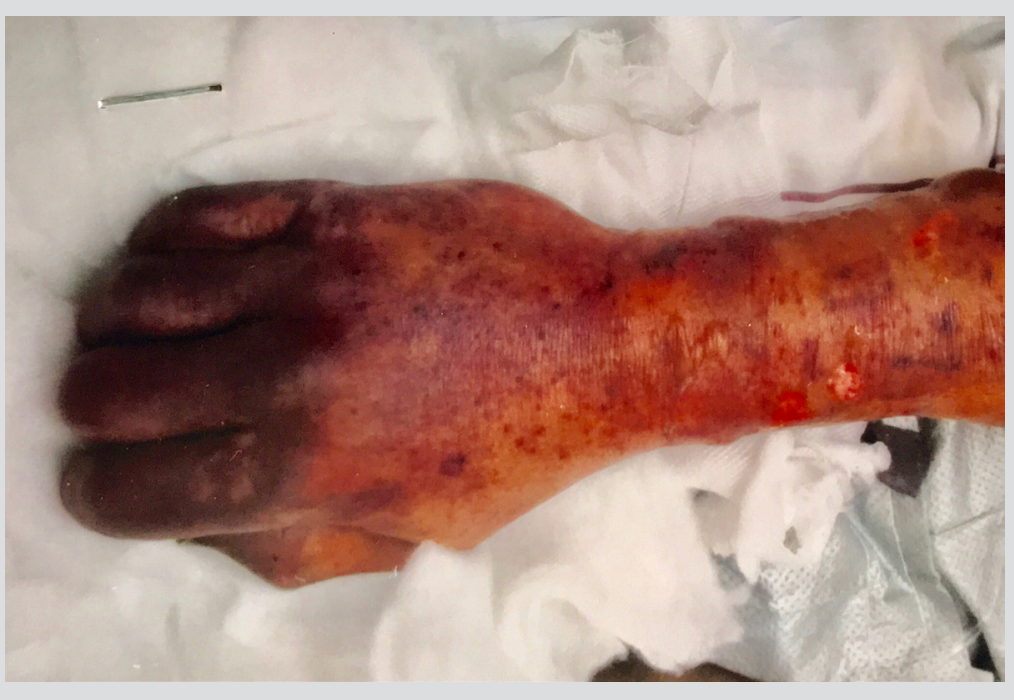

Figure 2. Patient's right forearm and hand after 1 week with unchanged purpura fulminans and emerging gangrene of the finger

On the 4th day of hospitalization, blood cultures yielded the Gram-negative bacillus Capnocytophaga canimorsus. Therefore, we added ciprofloxacin to the antibiotic regimen. The patient's history and clinical and laboratory examinations indicated no immunodeficiency, asplenia or alcohol abuse. While fever resolved and procalcitonin levels fell on the 8th day, CRP was rising and Candida albicans was isolated in repeated blood cultures. We began empirical treatment with fluconazole.

The patient developed progressive epidermolysis of the entire body. Signs of systemic infection persisted after 10 days of antibiotic treatment. In contrast, liver function, coagulation parameters and creatine kinase levels had normalized. CRP and procalcitonin levels rose again rapidly, and on day 11 the patient developed a temperature of $41^{\circ} \mathrm{C}$. In order to treat a possible hospital-acquired infection with methicillin-resistant Staphylococcus aureus, we added vancomycin to the antibiotic regime.

A CT scan of the chest showed atypical centrilobular and peribronchovascular consolidation, probably due to pneumonia. This was possibly associated with pulmonary aspergillosis, as Aspergillus fumigatus was detected in the patient's tracheal secretion, and we switched antimycotic treatment to voriconazole. Antibiotic treatment was changed to meropenem and penicillin G. Additionally, a CT scan of the abdomen showed complete splenic infarction, and all extremities turned gangrenous. As a cranial CT scan at this point showed signs of severe hypoxic cerebral oedema, a joint decision to de-escalate therapy was made together with relatives. The patient died after 16 days of treatment. 


\section{DISCUSSION}

Our report of a patient with fatal septic shock due to $C$. canimorsus describes several noteworthy features which may be important to clinical practice. C. canimorsus is a Gram-negative rod and facultative anaerobic bacterium that physiologically inhabits the oral cavity, especially of dogs and cats ${ }^{[1,2]}$. C. canimorsus infection is most frequently transmitted by dog bites ${ }^{[3]}$. Infections by C. canimorsus are generally rare, ranging from self-limiting, local skin infections to septic shock. Severe and fatal infections have been reported in patients with immunodeficiency, splenectomy or alcohol abuse ${ }^{[3,4]}$. Transmission by bite and frequent reports of immunodeficiency may indicate that a higher bacterial concentration and underlying preconditions are usually required to cause especially severe $C$. canimorsus infection. The occurrence of purpura fulminans is an early ominous sign of a progressively severe course ${ }^{[5]}$.

Our patient did not show any immunodeficiency, splenectomy or alcohol abuse. In addition, he only touched and was licked by his dog in the weeks prior to infection. Therefore, we assume there was a low bacterial concentration during transmission and no specific susceptibility to severe $C$. canimorsus infection. Despite this, the patient developed septic shock with fatal multiorgan failure. Additionally, he already had purpura fulminans on admission.

Very rarely, severe C. canimorsus infections without biting or scratching have been reported ${ }^{[6,7]}$. Only one patient had no immunodeficiency. C. canimorsus infection is fatal in approximately $25 \%$ of patients ${ }^{[3]}$. However, this high mortality rate is based on collections of case reports and case series which are limited by selection and publication bias and lack of differentiation between immunocompetent and immunodeficient patients. Purpura fulminans is also very rare and most commonly associated with severe infections ${ }^{[8]}$. Sepsis due to Neisseria meningitidis or Streptococcus spp., as well as Haemophilus influenza, Staphylococcus aureus and, very rarely, C. canimorsus is frequently complicated by purpura fulminans ${ }^{[8]}$.

What are the clinical implications of this case report? Pet owners with flu-like symptoms should urgently seek medical advice when their symptoms exceed those of a simple viral infection, which in this case were severe dyspnoea and petechiae. Physicians confronted with such patients should ask about contact with dogs and cats. They should consider $C$. canimorsus infections also in the presence of purpura fulminans and the absence of animal bites or scratches, and any immunodeficiency. In such cases, the clinician should immediately start empiric treatment with a penicillin in combination with a beta-lactam inhibitor until a definite diagnosis is established.

\section{REFERENCES}

1. Hore C. Important unusual infections in Australia: a critical care perspective. Crit Care Resusc 2001;3:262-272.

2. Janda JM, Graves MH, Lindquist D, Probert WS. Diagnosing Capnocytophaga canimorsus infections. Emerg Infect Dis 2006;12:340-342.

3. Butler T. Capnocytophaga canimorsus: an emerging cause of sepsis, meningitis, and post-splenectomy infection after dog bites. Eur J Clin Microbiol Infect Dis 2015;34:12711280.

4. Mantovani E, Busani S, Biagioni E, Venturelli C, Serio L, Girardis M. Purpura fulminans and septic shock due to Capnocytophaga canimorsus after dog bite: a case report and review of the literature. Case Rep Crit Care 2018;2018:7090268.

5. Pers C, Gahrn-Hansen B, Frederiksen W. Capnocytophaga canimorsus septicemia in Denmark, 1982-1995: review of 39 cases. Clin Infect Dis 1996;23:71-75.

6. Smeets NJ, Fijnheer R, Sebastian S, De Mast Q. Secondary thrombotic microangiopathy with severely reduced ADAMTS13 activity in a patient with Capnocytophaga canimorsus sepsis. Transfusion 2018;58:2426-2429.

7. Vignon G, Combeau P, Violette J, Cognée AS, Méglio S, Carrère F, et al. [A fatal septic shock due to Capnocytophaga canimorsus and review of literature]. Rev Med Interne 2018;39:820-823.

8. Colling ME, Bendapudi PK. Purpura fulminans: mechanism and management of dysregulated hemostasis. Transfus Med Rev 2018;32:69-76. 